\title{
Ultraintense Femtosecond Magnetic Nanoprobes Induced by Azimuthally Polarized Laser Beams
}

\author{
Manuel Blanco, ${ }^{\dagger}$ Ferran Cambronero, $^{\dagger}$ M. Teresa Flores-Arias, ${ }^{\dagger}$ Enrique Conejero Jarque, ${ }^{\ddagger}$ Luis $^{\text {Plaja, }}{ }^{\ddagger}$ \\ and Carlos Hernández-García* $*$ *๑) \\ †Grupo de Investigación Photonics4Life, Área de Óptica, Departamento de Física Aplicada, Universidade de Santiago de \\ Compostela, Santiago de Compostela, E-15782, Spain \\ *Grupo de Investigación en Aplicaciones del Láser y Fotónica, Departamento de Física Aplicada, University of Salamanca, E-37008, \\ Salamanca, Spain
}

\section{Supporting Information}

ABSTRACT: We report a novel scheme to generate laser-induced, ultrafast, intense (Tesla scale), spatially isolated, magnetic fields. Three-dimensional particle-in-cell simulations show that a femtosecond azimuthally polarized infrared vector beam, aimed at a conducting circular aperture, produces an intense axially polarized tip-shaped femtosecond magnetic field, extending over micrometer distances and being isolated from the electric field. Our results are backed up by an analytic model, demonstrating the underlying physics and guiding for optimal parameters. In particular, we find the conditions under which the magnetic nanoprobe is substantially

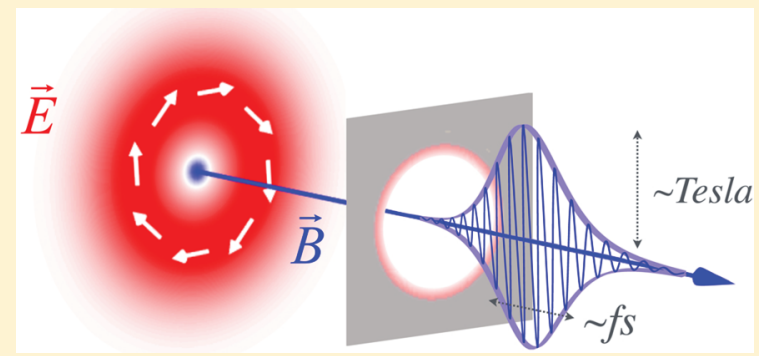
enhanced, reaching $4 \mathrm{~T}$ when driven by a $10^{11} \mathrm{~W} / \mathrm{cm}^{2}$ laser field, which reflects a selective enhancement by a factor of $\sim 6$. Our scheme offers a promising tool to control, probe, and tailor magnetic nanodomains in femtosecond time scales through pure magnetic interaction by using structured laser beams.

KEYWORDS: ultrafast magnetism, vector laser beams, femtosecond laser pulses, magnetic nanoprobes, femtomagnetism, nanophotonics, ultraintense magnetic field

$\mathrm{T}$ he development of new techniques to accurately control the spatial and temporal properties of materials is one of the most exciting challenges in current nanotechnology and a required step toward the next generation of electronic devices, magnetic storage, or photovoltaic materials, among others. In this context, current laser technology offers unique tools in the form of ultrashort pulses, as short as few femtoseconds ( $1 \mathrm{fs}=$ $\left.10^{-15} \mathrm{~s}\right)$ or even attoseconds $\left(10^{-18} \mathrm{~s}\right),^{1}$ that can be focused down to the nanometer scale ${ }^{2}$ and that can be structured in their angular momentum properties (polarization ${ }^{3}$ and orbital angular momentum ${ }^{4}$ ). Ultrashort laser sources have already shown great potential for applications in ultrafast magnetism. Since the pioneering work on ultrafast laser-induced demagnetization by Beaurepaire et al., ${ }^{5}$ fs laser pulses have been widely used in theoretical and experimental studies of femtomagnetism. $^{6-13}$ The magnetic effects in such short time scales appear not only due to thermal relaxation processes but also to coherent and almost instantaneous interactions between photons and spins. Such dynamics can only be tested using ultrashort laser pulses.

A remarkable feature of the interaction of laser pulses with magnetic materials is its strong dependence with the polarization. ${ }^{6,14,15}$ During recent years the development of vector beams-laser sources with structured polarization-has opened new avenues for magnetic laser-matter interaction. In particular, azimuthally polarized vector beams possess enor- mous potential to control pure magnetic interactions. ${ }^{16,17}$ Previous works have demonstrated the feasibility of azimuthally polarized laser beams to induce static magnetic fields, which can be enhanced toward few milli-Tesla through the use of nanoantennas ${ }^{18}$, with applications in nanoscale photoinduced force microscopy. ${ }^{19}$ With the development of ultrashort laser vector beams $\mathrm{s}^{20}$ we envision the opportunity to perform pure magnetic ultrafast interactions, including ultrafast demagnetization or spin precession. However, such interactions require the use of intense magnetic fields. Although in the last years Tesla-scale magnetic fields have been reported at the nanosecond and picosecond time scales, through laser-plasma interaction, ${ }^{21}$ or benefiting from transient thermoelectric currents in metals, ${ }^{22,23}$ to the best of our knowledge, there is no evidence of the generation of such intense, isolated, magnetic fields in the fs time scale.

In this work we propose a novel technique to generate intense, femtosecond longitudinal magnetic fields, spatially isolated from the electric field, using vector laser beams. We perform a complete analysis of the spatiotemporal properties of such magnetic pulses through the use of three-dimensional (3D) particle-in-cell (PIC) simulations and the development of an

Received: September 17, 2018

Published: December 7, 2018 
analytic model. In particular, we show that the longitudinally polarized magnetic field finds a maximum amplitude at the aperture axis, being isolated from the electric field and creating an ultrafast magnetic nanoprobe with a tip-like shape that extends over micrometer distances.

The electric field $(E)$ of an azimuthally polarized beam can be described in the paraxial approximation as a Laguerre-Gaussian mode $\left(\mathrm{LG}_{1,0}\right)$ without the azimuthal phase. ${ }^{24}$ Solving the Maxwell equations, one can obtain the corresponding magnetic field $(B) .{ }^{16}$ Thus, considering an azimuthally polarized laser beam propagating along the $x$ direction, the electromagnetic field obtained at the focal plane $(x=0)$ and expressed in cylindrical coordinates $(\rho, \varphi, x)$ reads as

$$
\begin{aligned}
\vec{E}(\vec{r})= & E_{0} \frac{\rho}{\omega_{0}} \mathrm{e}^{-\rho^{2} / \omega_{0}^{2}} \vec{e}_{\varphi} \\
\vec{B}(\vec{r})= & -\frac{E_{0}}{c} \frac{\rho}{\omega_{0}} \mathrm{e}^{-\rho^{2} / \omega_{0}^{2}} \vec{e}_{\rho}-2 \frac{E_{0}}{c} \frac{\lambda}{2 \pi \omega_{0}}\left[1-\frac{\rho^{2}}{\omega_{0}^{2}}\right] \mathrm{e}^{-\rho^{2} / \omega_{0}^{2}} \\
& \times \mathrm{e}^{i \pi / 2} \vec{e}_{x}
\end{aligned}
$$

where $E_{0}$ is the $E$ field amplitude and $\vec{e}_{\varphi}, \vec{e}_{\rho}$, and $\vec{e}_{x}$ are the unitary vectors in the cylindrical coordinate system. The beam waist is $w_{0} ; \lambda$ is the wavelength; and $c$ is the speed of light.

Figure 1a shows the interaction scheme considered: the azimuthally polarized beam is aimed perpendicularly to a metallic circular aperture. We perform numerical simulations

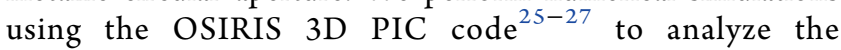
spatiotemporal behavior of the electromagnetic field with and
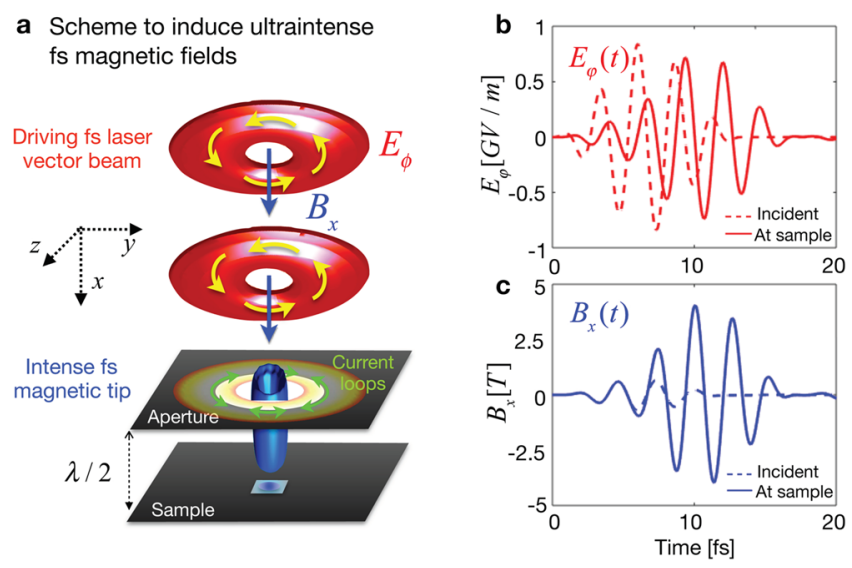

Figure 1. (a) Scheme to generate tip-shaped ultraintense ultrafast longitudinal $B$ fields. An azimuthally polarized laser beam (red) is aimed to a metallic circular aperture. The current loops (green arrows) induced at the edge of the aperture enhance the longitudinal $B$ field (blue) located at the singularity of the vector beam, resulting in a tipshaped ultraintense ultrafast $B$ field of micrometer scale. The dashed lines in panels (b) and (c) show the temporal profile of the incident azimuthal electric field $\left(E_{\varphi}\right)$ at $\rho=\rho_{0}$, and the incident longitudinal magnetic field $\left(B_{x}\right)$ on-axis $(\rho=0)$, respectively, detected at the focal plane $(x=0)$ and obtained through 3D PIC simulations. The solid lines represent the $E_{\varphi}$ and $B_{x}$ fields detected at a sample plane located at $x=$ $\lambda / 2$ when placing the circular aperture. When comparing the incident and apertured fields, whereas $E_{\varphi}$ remains similar, the apertured $B_{x}$ field is enhanced by a factor of $\sim 6$ (reaching $4 \mathrm{~T}$ ). Note that the $E_{\varphi}$ fields presented in (b) are detected at the spatial positions of maximum intensity for each case $-\rho=\rho_{0}$ for the incident field and $\rho=0.24 \mu \mathrm{m}$ for the apertured one-which is responsible for the temporal delay observed. without the metallic aperture. We have considered as an incident laser pulse the azimuthally polarized beam described by eqs 1 and 2, with waist $w_{0}=2.5 \mu \mathrm{m}$, a central wavelength of $\lambda=0.8 \mu \mathrm{m}$, and $E$ field amplitude of $0.86 \mathrm{GV} / \mathrm{m}$ (peak intensity of $10^{11} \mathrm{~W} /$ $\left.\mathrm{cm}^{2}\right)$ at the radius of maximum intensity $\left(\rho_{0}=w_{0} / \sqrt{2}\right)$. The temporal envelope is modeled as a $\sin ^{2}$ function of 4.8 fs full width at half-maximum in intensity. In order to implement the proposed scheme for the amplification of the $B$ field, we have considered thin gold circular apertures (density of $5.9 \times 10^{22}$ $\mathrm{cm}^{-3}$ and $50 \mathrm{~nm}$ in thickness) with different radii, $\rho_{\mathrm{A}}$, placed at the beam focus position $(x=0)$. Note that the maximum peak intensity of the laser pulse has been chosen to be below the damage threshold of the material of the aperture. In addition, we note that the laser pulse duration ( $4.8 \mathrm{fs}$ ) is well below the roomtemperature carrier relaxation time of $\mathrm{Au}(27.3 \mathrm{fs})$ and other metals such as $\mathrm{Ag}$ or $\mathrm{Cu}(\sim 36 \mathrm{fs}){ }^{28}$ Thus, the collisionless plasma simulated by the PIC code gives a reasonable description of the electronic currents induced by few-cycle laser pulses in the aperture, as it also takes into account metal edge effects and plasmon resonances.

The central outcome of our work is shown in Figure $1 \mathrm{~b}$ and c, where we present the $E_{\varphi}$ field at the radius of maximum intensity and the $B_{x}$ at $\rho=0$ of the incident (dashed lines) and apertured (solid lines) beams. Whereas the incident fields are shown at the focal plane $x=0$, the apertured fields are shown at a plane located at $x=\lambda / 2$. As a main result, we observe that the incident beam naturally induces an ultrafast $B_{x}$ field that oscillates at 0.37 $\mathrm{PHz}$, with a maximum amplitude of $0.7 \mathrm{~T}$ (dashed blue line in Figure 1c). Interestingly enough, when placing a gold circular aperture with radius equal to that of maximum intensity of the beam, $\rho_{\mathrm{A}}=\rho_{0}=1.78 \mu \mathrm{m}$, the $B_{x}$ amplitude is enhanced by a factor of $\sim 6$ with respect to the nonapertured case, reaching a peak value of $4 \mathrm{~T}$.

In Figure 2 we show the incident (left) and apertured (right) fields at the longitudinal (top) and transverse (bottom) planes obtained from our 3D PIC simulations. The spatial distributions are presented at the temporal instant when each field is maximum. When placing a gold aperture of radius $\rho_{\mathrm{A}}=\rho_{0}$, the $E_{\varphi}$ and $B_{x}$ fields are strongly focused, but, remarkably, both their longitudinal and transverse distributions remain separated (right side of Figure 2). Note that the radial magnetic field, $B_{\rho}$, is also spatially separated from $B_{x}$ (see Supporting Information).

Another remarkable feature of the $B$ field profile after the aperture is its narrow width in comparison with its longitudinal extension, forming a tip-shaped probe, as depicted in Figure $2 \mathrm{f}$. We have developed a simple model to characterize the spatial profile of this magnetic nanoprobe by analyzing the field induced by the alternate electric azimuthally polarized field in the circular plate. The model simplifies the picture assuming a thin metallic plate and an induced current with a narrow ring-shaped form (see Supporting Information). The current loop induces a $B_{x}$ field with maximum amplitude at the beam propagation axis, which can be described by $B_{x, 0}=\left|B_{x, 0}\right| \mathrm{e}^{\mathrm{i} \varphi_{0}}$, where

$$
\begin{aligned}
&\left|B_{x, 0}\left(\widetilde{\rho}_{0} ; \tilde{x}\right)\right| \propto \frac{1}{\lambda} \frac{\widetilde{\rho}_{0}^{2}}{\widetilde{\rho}_{0}^{2}+\tilde{x}^{2}}\left(4 \pi^{2}-\frac{1}{\widetilde{\rho}_{0}^{2}+\tilde{x}^{2}}\right)^{1 / 2} \\
& \varphi_{0}\left(\widetilde{\rho}_{0} ; \tilde{x}, t\right)= 2 \pi \sqrt{\widetilde{\rho}_{0}^{2}+\tilde{x}^{2}}-\arctan \left(2 \pi \sqrt{\widetilde{\rho}_{0}^{2}+\tilde{x}^{2}}\right) \\
&-\omega t
\end{aligned}
$$

with $\tilde{\rho}_{0}=\rho_{0} / \lambda$ and $\tilde{x}=x / \lambda$ being the normalized radial and longitudinal coordinates. We define the probe length, $l$, as the 

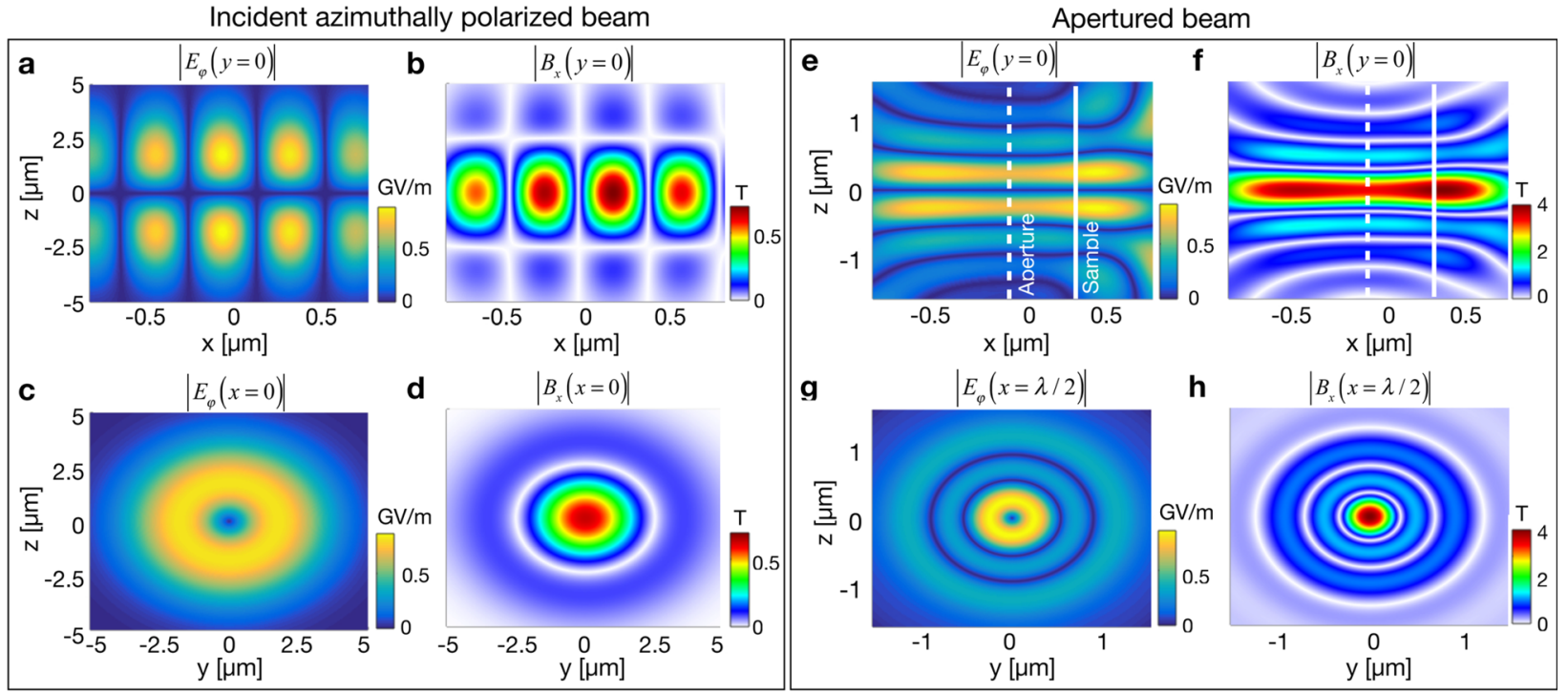

Figure 2. Spatial distributions of the $E_{\varphi}$ and $B_{x}$ fields carried by an azimuthally polarized laser pulse beam (left) without and (right) with a gold circular aperture of radius $\rho_{\mathrm{A}}=\rho_{0}=1.78 \mu \mathrm{m}$, placed at $x=0$. (a, e and b,f) Longitudinal spatial profiles of $E_{\varphi}$ and $B_{x}$ at $y=0$, respectively. (c and d) Transverse ones at the focal plane $x=0$ for the incident beam; ( $\mathrm{g}$ and $\mathrm{h}$ ) corresponding transverse profiles of the apertured $E_{\varphi}$ and $B_{x}$ fields at the sample plane $x=$ $\lambda / 2$, respectively. The aperture (white dashed) and sample (white solid) positions are indicated in (e) and (f). These spatial profiles are obtained through 3D PIC numerical simulations.

distance from the plate for which the $B$ field energy drops by a factor of 2 . Imposing this condition to the squared modulus of eq 3 , we find a simple scaling of the probe length with the radius of the current loop, $l \simeq 0.6 \rho_{0}$. Thus, the probe typically extends over distances exceeding a few $\lambda$, allowing for almost pure magnetic interactions close to the axis of a target plane located at micrometer distances from the aperture. From the practical point of view, a nearly uniform distribution of the $B_{x}$ field over the thickness of a potential target is desirable. Therefore, it becomes necessary that the phase of the field amplitude of the magnetic probe does not change rapidly along the target thickness dimension. We show in Figure 3 the probe extension as a function of the current loop radius, indicated by the gray

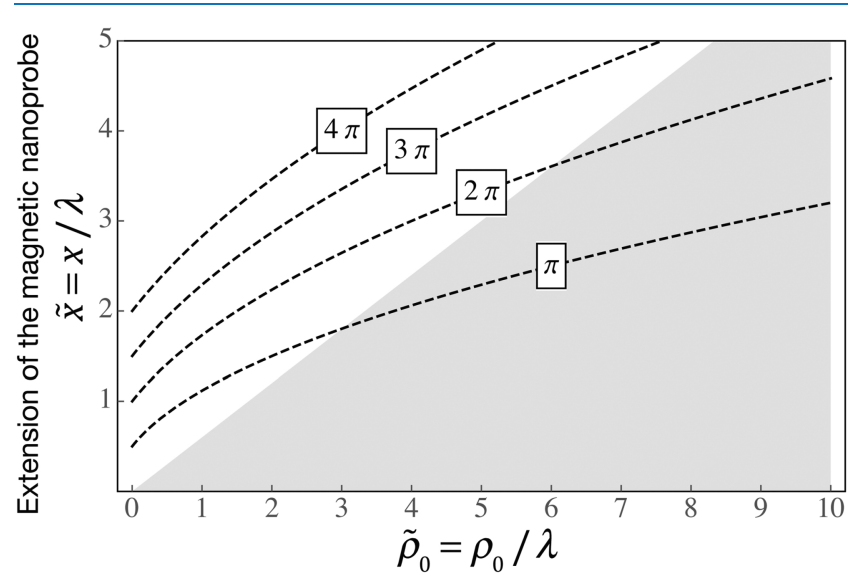

Radius of the current loop

Figure 3. Extension and phase of the magnetic nanoprobe as a function of the radius of the current loop induced in the aperture, obtained with the analytic model described by eqs 3 and 4 . The gray area shows the extension of the magnetic nanoprobe $\left(l \simeq 0.6 \rho_{0}\right)$ defined as the separation from the plate for which the $B_{x}$ field energy drops by a factor of 2 . The contoured dashed lines show the spatial phase shift of the $B_{x}$ field. area, and the spatial phase distribution, indicated by the contoured dashed lines. It is worth noting that for a loop radius of $3 \lambda$, the field energy is confined to a region extending over $\simeq 1.8 \lambda$, with a phase variation $<\pi$.

Our analytic model indicates that the electronic current induced at the edge of the aperture-and thus the induced $B_{x}$ field-reaches its maximum when the aperture radius matches that of the ring of maximum intensity of the vector beam, i.e., $\rho_{\text {A }}$ $=\rho_{0}$ (see Supporting Information). This is confirmed by the results from 3D PIC simulations shown in Figure 4(a), where we present the ratio $B_{x} / B_{x}^{0}$, being $B_{x}^{0}$ the incident field without the aperture, at $\rho=0$ for different aperture radii, measured at the sample plane $(x=\lambda / 2$, blue $)$ and at the spatial position of maximum $B_{x}$ (red). In addition, the aperture radius introduces a proportional temporal delay to the induced $B_{x}$ field, as can be observed in Figure 1(c). Movies in the Supporting Information show the temporal evolution of the buildup of the $E_{\varphi}$ and $B_{x}$ fields for different aperture radii.

At these high intensities it is interesting to increase not only the $B$ field amplitude but also the contrast between the $B$ and $E$ fields, which we define as $F=c\left|B_{x}\right| /\left|E_{\varphi}\right|$. In the 3D PIC simulations presented in Figures 1 and 2, we observe that, whereas the $B_{x}$ field is amplified when placing the aperture, the $E_{\varphi}$ field amplitude remains similar, resulting in an increased contrast $F$. This is a remarkable aspect of our scheme, as it allows increasing the $B$ field, avoiding high $E$ fields that could reach the damage threshold of the sample. Our simulations also show that the contrast between the maximum $B_{x}$ and $E_{\varphi}$ fields, $F_{\max }$, shown in Figure 4(b), is maximized for an aperture of radius $\rho_{\mathrm{A}}=\rho_{0}$.

Finally, in order to define the spatial region where the $B_{x}$ field can be considered as isolated, we present in Figure 4(c) the radial profile of the contrast between the $B_{x}$ and $E_{\varphi}$ fields, $F(\rho)=$ $c B_{x}(\rho) / E_{\varphi}(\rho)$, and in Figure $4(\mathrm{~d})$ that between the $B_{x}$ and $B_{\rho}$ fields, $F_{B}(\rho)=B_{x}(\rho) / B_{\rho}(\rho)$. Note that these curves are very similar for all aperture radii. From Figure $4(c)$ and (d) one can define a spatial region in which the $E_{\varphi}$ and/or the $B_{\rho}$ field can be neglected with respect to the $B_{x}$ field. Note that the size of this 

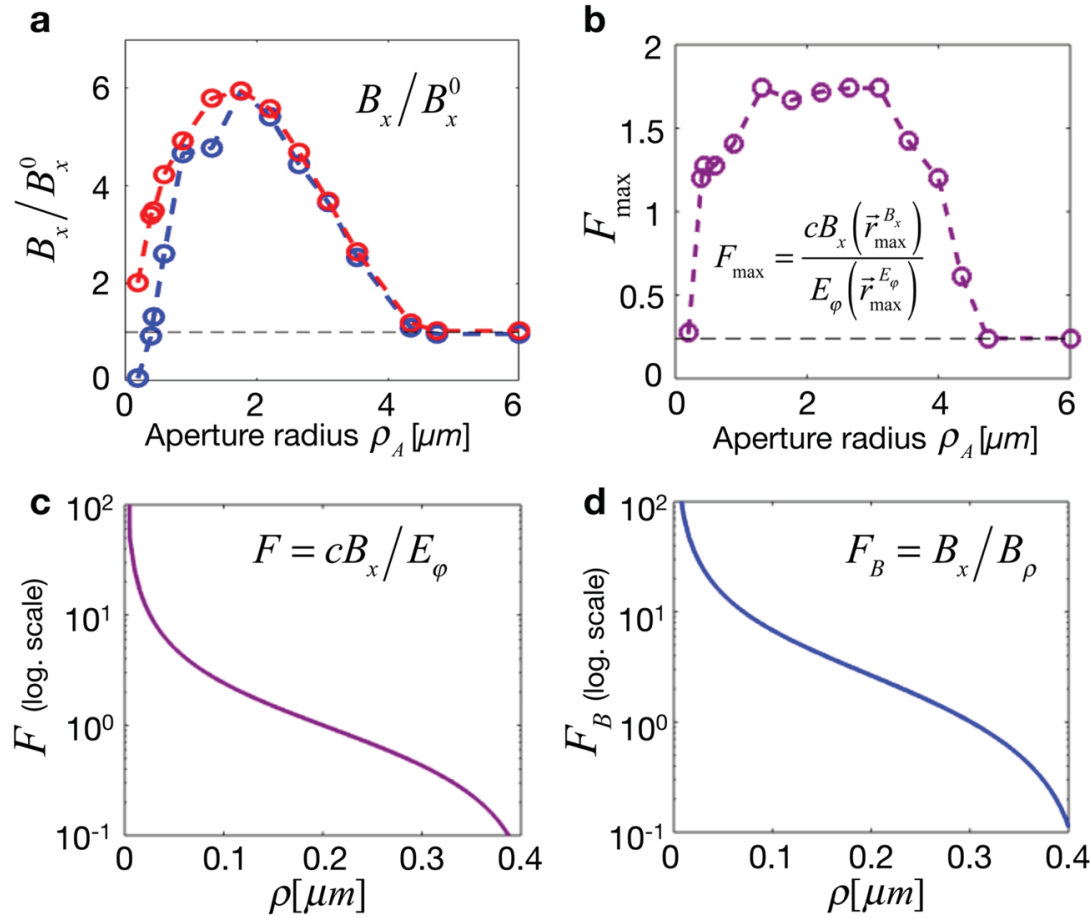

Figure 4. (a) Amplification of the $B_{x}$ field for different aperture sizes. The ratio $B_{x} / B_{x}^{0}$ at $\rho=0$ is presented for different aperture radii, where $B_{x}$ is measured at the sample $\left(x=\lambda / 2\right.$, blue) and at the spatial position where it is maximum (red), and $B_{x}^{0}$ is the incident field without the aperture. (b) Contrast between the maximum $B_{x}$ and $E_{\varphi}$ fields, i.e., $F_{\max }=c B_{x}\left(\vec{r}_{\max }^{B_{x}}\right) / E_{\varphi}\left(\vec{r}_{\max }^{E_{\varphi}}\right)$, where $\vec{r}_{\max }^{B_{x}}\left(\vec{r}_{\max }^{E_{\varphi}}\right)$ is the position where $B_{x}\left(E_{\varphi}\right)$ is maximum. The black dashed line indicates the value for the nonapertured case. The maximum amplification and contrast $F_{\max }$ take place for an aperture radius $\rho_{\mathrm{A}}=\rho_{0}$. (c) Radial profile of the contrast $F(\rho)=B_{x}(\rho) / E_{\varphi}(\rho)$ and (d) that of $F_{\mathrm{B}}(\rho)=B_{x}(\rho) / B_{\rho}(\rho)$, which allow us to define the spatial region in which the $B_{x}$ field is isolated from the $E_{\varphi}$ and $B_{\rho}$ fields.

region could be modified through the use of different beam waists $\left(w_{0}\right)$, keeping in mind that the optimal aperture for amplifying the $B_{x}$ field with respect to the $E_{\varphi}$ satisfies $\rho_{\mathrm{A}}=\rho_{0}$.

In conclusion, we present a scheme to generate ultraintense, ultrafast, isolated longitudinal magnetic fields from azimuthally polarized laser beams. The use of a thin circular aperture allows us to amplify the longitudinal magnetic field by a factor of $\sim 6$, reaching $4 \mathrm{~T}$ for an incident laser vector beam of $10^{11} \mathrm{~W} / \mathrm{cm}^{2}$, being thus comparable to the magnetic field amplitudes of the large bending magnets used in synchrotron facilities. We also have shown that the circular aperture enhances the contrast between the $B$ and $E$ fields, allowing for the isolation of the $B$ field in regions of several $\mathrm{nm}^{2}$. The $B$ field is found as a tipshaped probe whose length scales linearly with the aperture radius, allowing to implement pure magnetic interaction with a material target placed at micrometer distances from the plate. Thus, our scheme offers the possibility to tailor the spatial and temporal properties of the $B$ field through proper modifications of the incident laser beam and metallic aperture parameters.

Our findings present potential applications in different fields. On one hand, instead of controlling material magnetic properties with the $E$ field of a fs laser pulse, we envision the possibility of controlling the magnetic properties of materials with a fs $B$ field. For example, a fast magnetization reversal, which is of paramount importance for the realization of high-rate magnetic recording, could be triggered at the fs scale with $B$ fields of hundreds of T. One could also advance the possibility of generating attosecond isolated $B$ fields if shorter wavelength ultrashort vector beams ${ }^{20}$ were used. On the other hand, ultraintense, ultrafast $B$ nanoprobes can be applied to improve the capability to control the acceleration of charged particles in laser-plasma interaction schemes, ${ }^{29-31}$ to optimize the generation of coherent extreme-ultraviolet radiation through high harmonic generation, ${ }^{32}$ or to enhance the betatron oscillation amplitude in the generation of X-rays. ${ }^{33}$

\section{ASSOCIATED CONTENT}

\section{Supporting Information}

The Supporting Information is available free of charge on the ACS Publications website at DOI: 10.1021/acsphotonics.8b01312.

Detailed discussion of (I) the parameters of the 3D PIC simulations and the spatial and temporal profiles of the radial component of the $B$ field; (II) the temporal evolution of the spatial profile of the $E$ and $B$ fields propagating through different apertures; (III) a sensitivity analysis of the $B$ field amplification with the aperture shape; and (IV) the complete derivation of the analytic model that describes the spatial properties of the magnetic nanoprobe (PDF)

Temporal evolution of the spatial distribution of the $E_{\varphi}$ field for four different aperture radii (AVI)

Temporal evolution of the spatial distribution of the $B_{x}$ field for four different aperture radii (AVI)

Temporal evolution of the spatial distribution of the $B_{x}$ field for four different aperture thicknesses (AVI)

\section{AUTHOR INFORMATION}

\section{Corresponding Author}

*E-mail: carloshergar@usal.es. 


\section{ORCID $\odot$}

Carlos Hernández-García: 0000-0002-6153-2647

Notes

The authors declare no competing financial interest.

\section{ACKNOWLEDGMENTS}

The authors thank Luis López, Eduardo Martínez, and Victor J. Raposo for fruitful discussions. The authors thank the support from Ministerio de Economía y Competitividad (FIS201571933- REDT), Xunta de Galicia/ FEDER (ED431B 2017/64 and ED431E 2018/08), and Ministerio de Ciencia, Innovación y Universidades (EQC2018-0041 17-P). C.H.-G. acknowledges support from a 2017 Leonardo Grant for Researchers and Cultural Creators, BBVA Foundation. C.H.-G., E.C.J., and L.P. acknowledge support from Junta de Castilla y León (SA046U16, SA287P18) and Ministerio de Economía y Competitividad (FIS2016-75652-P). M.B. is funded by the FPU grant program of MECD. The authors acknowledge the OSIRIS Consortium, consisting of UCLA and IST (Lisbon, Portugal) for the use of OSIRIS, for providing access to the OSIRIS framework.

\section{REFERENCES}

(1) Krausz, F.; Ivanov, M. Attosecond physics. Rev. Mod. Phys. 2009, 81, 163-234.

(2) Popmintchev, T.; et al. Bright Coherent Ultrahigh Harmonics in the keV X-ray Regime from Mid-Infrared Femtosecond Lasers. Science 2012, 336, 1287-1291.

(3) Huang, P.-C.; et al. Polarization control of isolated high-harmonic pulses. Nat. Photonics 2018, 12, 349-357.

(4) Hernández-García, C.; Picón, A.; San Román, J.; Plaja, L. Attosecond Extreme Ultraviolet Vortices from High-Order Harmonic Generation. Phys. Rev. Lett. 2013, 111, 083602.

(5) Beaurepaire, E.; Merle, J.-C.; Daunois, A.; Bigot, J.-Y. Ultrafast Spin Dynamics in Ferromagnetic Nickel. Phys. Rev. Lett. 1996, 76, $4250-4253$.

(6) Kimel, A. V.; Kirilyuk, A.; Usachev, P. A.; Pisarev, R. V.; Balbashov, A. M.; Rasing, T. Ultrafast non-thermal control of magnetization by instantaneous photomagnetic pulses. Nature 2005, 435, 655-657.

(7) Koopmans, B.; Malinowski, G.; Dalla Longa, F.; Steiauf, D.; Fähnle, M.; Roth, T.; Cinchetti, M.; Aeschlimann, M. Explaining the paradoxical diversity of ultrafast laser-induced demagnetization. Nat. Mater. 2010, 9, 259.

(8) Bigot, J.-Y.; Vomir, M.; Beaurepaire, E. Coherent ultrafast magnetism induced by femtosecond laser pulses. Nat. Phys. 2009, 5, 515-520.

(9) Boeglin, C.; Beaurepaire, E.; Halté, V.; López-Flores, V.; Stamm, C.; Pontius, N.; Dürr, H. A.; Bigot, J.-Y. Distinguishing the ultrafast dynamics of spin and orbital moments in solids. Nature 2010, 465, 458.

(10) Rudolf, D.; et al. Ultrafast magnetization enhancement in metallic multilayers driven by superdiffusive spin current. Nat. Commun. 2012, 3, 1037.

(11) Turgut, E.; La-o vorakiat, C.; Shaw, J. M.; Grychtol, P.; Nembach, H. T.; Rudolf, D.; Adam, R.; Aeschlimann, M.; Schneider, C. M.; Silva, T. J.; Murnane, M. M.; Kapteyn, H. C.; Mathias, S. Controlling the Competition between Optically Induced Ultrafast Spin-Flip Scattering and Spin Transport in Magnetic Multilayers. Phys. Rev. Lett. 2013, 110, 197201.

(12) Chen, C.; et al. Distinguishing attosecond electron-electron scattering and screening in transition metals. Proc. Natl. Acad. Sci. U. S. A. 2017, 114, E5300-E5307.

(13) Tengdin, P.; You, W.; Chen, C.; Shi, X.; Zusin, D.; Zhang, Y.; Gentry, C.; Blonsky, A.; Keller, M.; Oppeneer, P. M.; Kapteyn, H. C.; Tao, Z.; Murnane, M. M. Critical behavior within 20 fs drives the outof-equilibrium laser-induced magnetic phase transition in nickel. Sci. Adv. 2018, 4, eaap9744.
(14) Stanciu, C. D.; Hansteen, F.; Kimel, A. V.; Kirilyuk, A.; Tsukamoto, A.; Itoh, A.; Rasing, T. All-Optical Magnetic Recording with Circularly Polarized Light. Phys. Rev. Lett. 2007, 99, 047601.

(15) Fan, T.; et al. Bright circularly polarized soft X-ray high harmonics for X-ray magnetic circular dichroism. Proc. Natl. Acad. Sci. U. S. A. 2015, 112, 14206-14211.

(16) Veysi, M.; Guclu, C.; Capolino, F. Vortex beams with strong longitudinally polarized magnetic field and their generation by using metasurfaces. J. Opt. Soc. Am. B 2015, 32, 345-354.

(17) Veysi, M.; Guclu, C.; Capolino, F. Focused azimuthally polarized vector beam and spatial magnetic resolution below the diffraction limit. J. Opt. Soc. Am. B 2016, 33, 2265-2277.

(18) Guclu, C.; Veysi, M.; Capolino, F. Photoinduced Magnetic Nanoprobe Excited by an Azimuthally Polarized Vector Beam. ACS Photonics 2016, 3, 2049-2058.

(19) Zeng, J.; Huang, F.; Guclu, C.; Veysi, M.; Albooyeh, M.; Wickramasinghe, H. K.; Capolino, F. Sharply Focused Azimuthally Polarized Beams with Magnetic Dominance: Near-Field Characterization at Nanoscale by Photoinduced Force Microscopy. ACS Photonics 2018, 5, 390-397.

(20) Hernández-García, C.; Turpin, A.; Román, J. S.; Picón, A.; Drevinskas, R.; Cerkauskaite, A.; Kazansky, P. G.; Durfee, C. G.; nigo, J.; Sola, I. Extreme ultraviolet vector beams driven by infrared lasers. Optica 2017, 4, 520-526.

(21) Sandhu, A. S.; Dharmadhikari, A. K.; Rajeev, P. P.; Kumar, G. R.; Sengupta, S.; Das, A.; Kaw, P. K. Laser-Generated Ultrashort Multimegagauss Magnetic Pulses in Plasmas. Phys. Rev. Lett. 2002, $89,225002$.

(22) Tsiatmas, A.; Atmatzakis, E.; Papasimakis, N.; Fedotov, V.; Luk'yanchuk, B.; Zheludev, N. I.; de Abajo, F. J. G. Optical generation of intense ultrashort magnetic pulses at the nanoscale. New J. Phys. 2013, 15, 113035.

(23) Vienne, G.; Chen, X.; Teh, Y. S.; Ng, Y. J.; Chia, N. O.; Ooi, C. P. Novel layout of a bi-metallic nanoring for magnetic field pulse generation from light. New J. Phys. 2015, 17, 013049.

(24) Zhan, Q. Cylindrical vector beams: from mathematical concepts to applications. Adv. Opt. Photonics 2009, 1, 1-57.

(25) Fonseca, R. A.; Silva, L. O.; Tsung, F. S.; Decyk, V. K.; Lu, W.; Ren, C.; Mori, W. B.; Deng, S.; Lee, S.; Katsouleas, T.; Adam, J. C. OSIRIS: A Three-Dimensional, Fully Relativistic Particle in Cell Code for Modeling Plasma Based Accelerators; Computational Science-ICCS 2002: Berlin, Heidelberg, 2002; pp 342-351.

(26) Fonseca, R. A.; Martins, S. F.; Silva, L. O.; Tonge, J. W.; Tsung, F. S.; Mori, W. B. One-to-one direct modeling of experiments and astrophysical scenarios: pushing the envelope on kinetic plasma simulations. Plasma Phys. Controlled Fusion 2008, 50, 124034.

(27) Fonseca, R. A.; Vieira, J.; Fiuza, F.; Davidson, A.; Tsung, F. S.; Mori, W. B.; Silva, L. O. Exploiting multi-scale parallelism for large scale numerical modelling of laser wakefield accelerators. Plasma Phys. Controlled Fusion 2013, 55, 124011.

(28) Gall, D. Electron mean free path in elemental metals. J. Appl. Phys. 2016, 119, 085101.

(29) Vieira, J.; Martins, S. F.; Pathak, V. B.; Fonseca, R. A.; Mori, W. B.; Silva, L. O. Magnetic Control of Particle Injection in Plasma Based Accelerators. Phys. Rev. Lett. 2011, 106, 225001.

(30) Rassou, S.; Bourdier, A.; Drouin, M. Influence of a strong longitudinal magnetic field on laser wakefield acceleration. Phys. Plasmas 2015, 22, 073104.

(31) Korneev, P.; Tikhonchuk, V.; d'Humieres, E. Magnetization of laser-produced plasma in a chiral hollow target. New J. Phys. 2017, 19, 033023.

(32) Milošević, D. B.; Starace, A. F. Magnetic-Field-Induced Intensity Revivals in Harmonic Generation. Phys. Rev. Lett. 1999, 82, 26532656.

(33) Zhang, Z. M.; Zhang, B.; Hong, W.; Yu, M. Y.; Deng, Z. G.; Teng, J.; He, S. K.; Gu, Y. Q. Enhanced $\mathrm{x}$-rays from resonant betatron oscillations in laser wakefield with external wigglers. Plasma Phys. Controlled Fusion 2016, 58, 105009. 